\title{
Dificultad de los verbos fraseológicos en inglés para los hispanohablantes
}

Sonia Calderón

Universidad Nacional, Costa Rica

\section{RESUMEN}

El artículo of rece un análisis lingüístico de la dificultad que presentan los verbos fraseológicos en inglés para los hispanohablantes, teniendo en cuenta su estructura sintáctica, su función semántica y pragmática y su aspecto fonológico. Como complemento, se propone una guía para la enseñanza de estos verbos y su práctica en clase.

\section{Abstract}

This article presents a linguistic analysis of the difficulty of English phrasal verbs for Spanish speakers, including their syntactic structure, their semantic and pragmatic function, and phonological aspects. Guidelines are also given for teaching and practicing these verbs.

\section{Introducción}

Este artículo of rece un análisis lingüístico de la dificultad que presentan los verbos fraseológicos en inglés para los hispanohablantes, teniendo en cuenta su estructura sintáctica, su función semántica y pragmática y su aspecto fonológico. Además se propone una guía para la enseñanza de estos verbos y su práctica en la clase de inglés como lengua extranjera. 
Debido a las necesidades actuales, donde la globalización trae implicaciones socioeconómicas, culturales y políticas, el uso del inglés como lengua extranjera se ha convertido en una necesidad para todas las personas. Su influencia en la cultura costarricense por medio de libros, televisión, películas, Internet y de algunas compañías como Intel, ha hecho del inglés una necesidad prioritaria tanto en el desarrollo personal como colectivo en Costa Rica. Hace algunos años, el sistema educativo público costarricense incorporó la enseñanza del inglés en el currículo de la escuela primaria. En secundaria ya existía desde hace varias décadas. También consciente de la importancia de este idioma en nuestro ámbito, recientemente el Ministerio de Educación Pública creó los colegios bilingües públicos en los que los estudiantes reciben diez lecciones semanales del idioma extranjero. Con esto se busca preparar mejor a los jóvenes para un mundo cada vez más competitivo en todas las áreas del desarrollo. En la enseñanza del inglés como lengua extranjera, los docentes deben tener en cuenta todas estas características de nuestro ámbito para que los educandos se comuniquen de la mejor manera posible. El docente debe proveer actividades y prácticas apropiadas para lograr este propósito, sobre todo al enseñar ciertas estructuras de difícil comprensión y uso.

En el campo de la lingüística aplicada se ha examinado ampliamente la influencia de la lengua materna en la adquisición de un segundo idioma. En algunos casos se ve esa influencia como negativa para el proceso de aprendizaje; en otros casos se ha visto que la lengua materna puede ser beneficiosa para la persona, especialmente si es adolescente $o$ adulta.

La teoría de análisis comparativo de la adquisición de un segundo idioma proponía que las dificultades de un individuo para adquirir otro idioma se podían prever al comparar las estructuras de la lengua materna y la lengua extranjera. Dulay y otros han señalado lo siguiente:

For several decades, linguists and teachers assumed that most second language learners' errors resulted from differences 
between the first and second languages. Studies show that only $5 \%$ of the grammatical errors children make and at most $20 \%$ of the ones adults make can be traced to crossover from the first language ${ }^{1}$.

Muchas investigaciones han demostrado que no necesariamente aquellas estructuras que difieren en los dos idiomas son las que causan problemas a los estudiantes. Además, algunos afirman que no existe un método eficiente para comparar la estructura de dos idiomas.

Sin embargo, un aspecto de la sintaxis inglesa que se les dificulta a los hispanoparlantes es el uso de los verbos fraseológicos o de dos palabras. Estas estructuras se usan con mucha frecuencia en inglés, tanto en el lenguaje hablado como en el escrito. Es importante que los estudiantes de inglés como lengua extranjera estén familiarizados con ellos, los entiendan y los puedan usar en el contexto apropiado. La dificultad de estos verbos para los que hablan español radica en la historia lingüística del inglés y del español. Siendo el inglés de origen germánico, como el alemán y el holandés, contiene este tipo de estructura. Muy pocos idiomas de origen no germánico la contienen. Por lo tanto, los estudiantes de inglés como segundo idioma o como idioma extranjero encontrarán dichos verbos extraños y difíciles.

Estos verbos están formados por un verbo y una partícula (o en algunos casos por dos partículas). Las partículas pueden ser preposiciones o adverbios, que aunque similares, actúan en forma diferente dentro de los verbos fraseológicos. Estas estructuras son parte tan importante del inglés coloquial que nadie podría hablar o entender una conversación corriente sin el conocimiento de ellas. Las personas, cuya lengua materna no es el inglés, tienen la tendencia de usar verbos simples en una conversación cotidiana, donde lo más apropiado sería un verbo fraseológico.

1. Marina Dulay, Heidi Burt y Stephen Krashen, Language Two (Nueva York: Oxford University Press, 1983) 5. 
En inglés, el uso del verbo simple podría verse como pedante y extraño en un contexto informal. Por ejemplo, Could you extinguish your cigarette?, suena demasiado formal y artificial y se prefiere el correspondiente Could you put out your cigarette?

Los verbos fraseológicos constituyen una categoría muy productiva dentro del léxico en inglés, tanto que no podemos adelantar qué verbo se unirá con qué partícula para formar un nuevo verbo fraseológico. Aun más, en algunos casos no podríamos predecir cuál será su significado, puesto que muchos de éstos son usados en forma idiomática. Tal y como lo indican Celce-Murcia y Larsen-Freeman:

Certain particles such as up, down, on, off and back can readily form phrasal verbs by combining with common verbs such as be, come, go, do, make and give. Phrasal verbs are a highly productive lexical category in English ${ }^{2}$.

La creación de Internet y su acceso a un público cada vez mayor han producido muchos nuevos verbos fraseológicos. Por ejemplo, tenemos scroll down, que significa llevar el cursor hacia abajo en la página. Un segundo ejemplo es click on, que quiere decir marcar una conexión con el ratón para entrar a ella. Además tenemos log on, que se usa para abrir una cuenta de correo electrónico y su contraparte log off, que utilizamos para indicar que deseamos cerrar nuestro correo. También tenemos zoom in (ver la pantalla más pequeña) y zoom out (ver la pantalla más grande). Además, los padres de familia pueden adquirir un programa especial para que sus hijos pequeños no tengan acceso a páginas inapropiadas y entonces se puede decir "block out a site or web page", que significa "no permitir el acceso a cierto sitio o página".

2. Marianne Celce-Murcia y Diane Larsen-Freeman, The Grammar Book (Rowley: Newbury House, 1983) 266. 


\section{Materiales didácticos utilizados en Costa Rica}

$\mathrm{Al}$ examinar varios textos que se usan para la enseñanza del inglés como lengua extranjera en Costa Rica, vemos que no se les da mayor importancia a los verbos fraseológicos y sólo los presentan en forma aislada en una unidad. Por ejemplo, el texto Interchange 2, que se usa en secundaria y a nivel universitario (Instituto Tecnológico de Costa Rica, Universidad Nacional), sólo incluye una unidad en un total de quince unidades donde se habla de dicha estructura. Introduce una serie de ocho verbos y un ejercicio mecánico con ellos ${ }^{3}$. Después no se vuelven a mencionar o repasar. El texto Up Close 4. English for Global Communication no tiene una sola unidad con verbos fraseológicos ${ }^{4}$. El texto Learning English. Supplementary Material for Costa Rican Students, que se usa en la educación secundaria pública, tampoco tiene en cuenta dichos verbos como parte de sus contenidos 5 .

\section{Aspectos sintácticos}

Un primer aspecto sintáctico es que precisamente porque no se pueden comparar con una estructura en particular en el idioma español, los verbos fraseológicos en inglés presentan gran dificultad para los hispanoparlantes, tanto a la hora de entender su uso como al usarlos apropiadamente según el contexto. Las partículas dan al verbo un significado totalmente distinto al del verbo por sí solo. El significado no puede predecirse del significado del verbo y su partícula por separado. Según Quirk y Greenbaum:

3. Jack C. Richards, Interchange. English for International Communication 2 (Nueva York: Cambridge University Press, 1991) 25.

4. Isobel Rainey de Díaz y otros, Up Close 4. English for Global Communication (Boston: Heinle and Heinle, 2002) xii.

5. Leonor Cabrera y Patricia Córdoba, Leaming English. Supplementary Material for Costa Rican Students (México D.F.: McGraw-Hill Interamericana, 1994). 
Phrasal verbs vary in the extent to which the combination preserves the individual meanings of verb and particle. In instances like give in, catch on and turn up, it is clear that the meaning of the combination cannot be predicted from the meanings of the verb and particle in isolation ${ }^{6}$.

Otro ejemplo, el verbo call significa "llamar, gritar", pero si tomamos este verbo y le agregamos la partícula off, significa "aplazar" o "suspender", como por ejemplo en They called off the meeting (Cancelaron la reunión). Otro ejemplo es el verbo call up, que significa "llamar por teléfono", como en la oración I have to call up George (Tengo que llamar a Jorge por teléfono). Este fenómeno lingüístico hace que los hispanoparlantes tengan problemas en entender el uso y el significado de estas estructuras. Tales verbos también pueden estar compuestos por un verbo seguido de dos preposiciones, como por ejemplo run out of, que significa "acabarse", como en We are running out of gas (Se nos está acabando la gasolina). Otro ejemplo es get along with, como en la oración John and I get along well (John y yo nos llevamos bien).

Los verbos fraseológicos pueden clasificarse de tres formas: siempre transitivos, siempre intransitivos, o a veces el mismo verbo puede ser transitivo o intransitivo con un significado diferente. Por ejemplo, el verbo put on (ponerse) es siempre transitivo, mientras que el verbo get up (levantarse) es siempre intransitivo. Sin embargo, el verbo take off puede ser transitivo en la oración I take off my coat (Me quito el abrigo) y puede ser intransitivo en The plane takes off at $8(\mathrm{El}$ avión sale a las 8). Un segundo ejemplo es work out. En la oración They have to work out their differences, el verbo es transitivo y significa que ellos tienen que arreglar sus diferencias. Mientras que en They work out at the gym, el verbo es intransitivo y significa que ellos hacen ejercicio en el gimnasio.

6. Randolph Quirk y Sydney Greenbaum, A Concise Grammar of Contemporary English (Nueva York: Harcourt Brace, Jovanovich, 1973) 348. 
Otro cambio sintáctico que sufren estos verbos y que se debe tener en cuenta a la hora de presentarlos en una lección es que pueden ser clasificados como "separables" e "inseparables". Los separables permiten que el verbo y la partícula se separen al usar el complemento directo o su correspondiente pronombre, como por ejemplo They put off the meeting (Ellos pospusieron la reunión), que se puede decir They put the meeting off o They put it off. Cabe destacar que en los verbos separables si se usa un pronombre como sustituto del complemento directo, éste siempre debe colocarse entre el verbo y su partícula. Por lo tanto, la oración * They put off it no es aceptable. Como hay muchos casos donde el verbo y la partícula de los verbos fraseológicos se separan, los estudiantes preguntan cuándo debe suceder esto. Estos verbos pueden separarse sólo si el complemento directo es pronominal, principio cuyo uso es muy útil para presentar estos verbos a los estudiantes. Tal y como lo señala Celce-Murcia:

If the direct object is long (phonologically multi-syllabic) or with complex grammar, the particle must be right next to the verb ${ }^{7}$.

Otra forma de ver este principio es decir que si el complemento directo es corto y simple, es más factible que el verbo y sus partículas se separen. De hecho, este principio de Susan Ulm ha sido confirmado por un estudio de Cumming, Dwyer y Snow, donde analizaron seiscientos cincuenta casos de la separación de la partícula del verbo. Encontraron que el tamaño y la complejidad del complemento directo son las mejores bases para predecir la separación de la partícula del verbo ${ }^{8}$.

Otro aspecto sintáctico que cabe destacar es que muchas de estas estructuras pueden transformarse en adjetivos y en sustantivos. El verbo sit in expresa la idea de que un supervisor o colega visita

7. Celce-Murcia, 266.

8. S. Cumming y otros, "Figuring Particle Movement Out and Figuring Out Particle Movement" (traba jo inédito del curso Linguisitics 253, UCLA, segundo semestre, 1982). 
una clase. Al sustantivarse en a sit-in su significado es protesta o paro laboral. Otro ejemplo es break down, que se transforma en breakdown, como por ejemplo nervous breakdown, que expresa una crisis nerviosa. Un tercer ejemplo es el verbo look out, que quiere decir tener cuidado. Al sustantivarse lookout se usa para describir un lugar desde el cual se puede apreciar el paisaje, un mirador.

También algunos de estos verbos pueden actuar como adjetivos. En la frase a make-up test (examen de reposición), el verbo make up modifica al sustantivo test. El verbo run away significa "huir", "escaparse", pero en la frase runaway plot significa "plan de escape". Además pueden formar adjetivos participios, como por ejemplo a shut-down factory y de gerundio, como por ejemplo a cooling-off period.

Los verbos no separables se caracterizan porque el verbo nunca puede ser separado de su preposición o adverbio, como por ejemplo run into (encontrarse con alguien inesperadamente), como en la oración I ran into an old high school classmate yesterday (Me encontré con un compañero del colegio ayer). La oración I ran an old high school classmate into yesterday no es gramatical. Otro caso es stick with (seguir con), como por ejemplo en We have to stick with the old accounting system (Debemos proseguir con el antiguo sistema de contabilidad). Algunas veces se usa un verbo fraseológico más una preposición. El complemento directo va después de la preposición, como en He is looking forward to his vacation.

\section{Aspectos semánticos de los verbos fraseológicos}

El verbo fraseológico es una unidad semántica, ya que si separamos el significado del verbo y el significado de la correspondiente partícula por aparte, no tienen sentido en la mayoría de los casos. Por ejemplo, Dagut y Laufer dividieron este tipo de verbos en tres categorías por sus diferencias semánticas ${ }^{9}$ :

9. M. Dagut y B. Laufer, "Avoidance of phrasal verbs: A case for contrastive analysis", Studies in Second Language Acquisition, 7:73-9, 1985. 
1. Los verbos fraseológicos literales, donde la partícula retiene su significado literal adverbial, como sit down (sentarse) y hand out (entregar).

2. Los verbos fraseológicos completivos, donde la partícula indica la acción completada, como por ejemplo la partícula up en la oración He tore up the paper (Él rompió el papel en pedazos pequeños).

3. Los verbos fraseológicos figurativos, donde no hay forma sistemática de una asociación semántica del verbo y la partícula: She looked up the information (Ella trató de encontrar la información). Otros verbos son give in (rendirse) y turn up (arribar, surgir, aparecer). Esta última categoría es la más difícil de manejar para los estudiantes.

Así, tomemos como ejemplo drop by, donde drop significa "caerse" y by significa "por". La expresión drop by quiere decir visitar a alguien sin previos planes. Vemos que el significado de la unidad semántica es diferente al verbo y la preposición por separado.

En el caso de que haya un verbo frasealógico y un correspondiente verbo para expresar una idea, existe una diferencia semántica importante entre el verbo fraseológico y su correspondiente verbo, como por ejemplo invent y make up, los cuales pueden ser traducidos como "inventar". Hay preferencia por el uso del verbo fraseológico, tanto en el lenguaje oral como escrito. Un segundo ejemplo lo constituye el verbo stand up y su contraparte rise, que quieren decir ponerse de pie. En la práctica, el verbo rise sólo se usa para expresar ese acto directivo en situaciones formales como en una corte.

En el lenguaje informal sobre todo, los verbos fraseológicos son mucho más frecuentes que su correspondiente contraparte. Es usual oír the epidemic broke out in the southern states y no the epidemic began in the southern states (la epidemia empezó en los estados del sur). Un segundo ejemplo sería cut out some pictures from these magazines, que nunca se expresaría como remove some pictures from these magazines using the scissors (recorte unas fotos de estas 
revistas). Como podemos observar en distintos medios, estos verbos compuestos se usan más que su contraparte.

Al tratar este tema en un ámbito educativo, se debe explicar el verbo en un contexto real y no sólo dar su definición, ya que puede que no quede bien claro. Por ejemplo, en español nosotros llenamos un vaso con agua, llenamos una solicitud de empleo y llenamos los espacios en blanco en un examen. En inglés, you fill up a glass of water, you fill out an application form y you fill in the blanks on a test. Además, existe la expresión fill in for, que significa "sustituir a alguien en el trabajo". Todas estas diferencias deben tenerse en cuenta a la hora de enseñar a los estudiantes el uso de esta estructura, la cual debe ser presentada en un contexto real y nunca en una lista con su correspondiente significado. Una posibilidad es darle a los estudiantes un párrafo para completar con una lista de palabras. Se escribe el verbo fill in subrayado para que entiendan su significado sin necesidad de traducirlo. Por ejemplo:

Fill in the blanks with the following words:

sunny sandals weather

We went to the beach last week. The was warm. It was We wore shorts and

Luego se les da una hoja para completar con el verbo fill out subrayado. Por ejemplo:

Fill out this form:

Last name First name

Date of birth:

Place of birth:

Address:

Phone number: 
Con ejercicios como éstos los estudiantes entienden que para completar una información dada se usa fill in y fill out para dar información específica no dada.

Otra posibilidad es asignar a los estudiantes que escriban una oración o párrafo usando put off, look over y hand out y el resultado sería, We have to put off the meeting until next week because we haven't looked over the final copy of the documents we have to hand out to the committe (Tenemos que posponer la reunión hasta la semana próxima porque no hemos revisado la copia final de los documentos que debemos entregar a los miembros del comité). Otra forma de presentar los verbos es en un diálogo.

A: Are we having the meeting next Friday?

B: I'm afraid we have to put it off. I haven't looked over the papers we have to hand out.

A: Fine, I'll call up the members of the committee to let them know.

La idea consiste en siempre tratar de contextualizar el uso de los verbos fraseológicos al presentarlos en un contexto y no simplemente en forma de lista con su respectivo significado, tal y como aparecen en una gran cantidad de textos.

\section{Aspectos pragmáticos}

El lenguaje tiene una función pragmática, por lo que se debe tener en cuenta la fuerza del acto del habla, dependiendo de si se usa un verbo fraseológico u otro verbo con un significado similar. La expresión knock it off (deje de hacer eso) conlleva mucha más fuerza ilocutiva que stop doing that.

Por otro lado se puede decir, The guests have to dress up for the reception (Los invitados deben usar ropa formal para la recepción) y no The guests have to wear special clothes for the reception. $\mathrm{O}$ bien, 
The textile company laid off 300 employees (La compañía textil despidió a 300 empleados) y no The textile company terminated the employment of 300 people. Si estamos hablando de medios de transporte, no decimos, Board bus 34 at the corner and exit at the terminal sino Get on bus 34 at the corner and get off at the terminal (Súbase al autobús 34 en la esquina y bájese en la terminal).

Todas estas diferencias de orden pragmático deben tenerse en cuenta, tanto en la preparación de materiales de enseñanza como en la práctica que se les proporcione a los estudiantes. Para facilitar la comprensión de los verbos get on y get off, el profesor puede llevar al aula un cajón que representa un autobús o tren. Un estudiante sube al cajón y dice, I get on the bus. Otro estudiante dice, He gets on the bus. Luego se baja del cajón y dice, He gets off the bus. Luego toda la clase puede crear un diálogo para usar estos dos verbos.

\section{Aspectos fonológicos}

En el aspecto fonológico, cuando el verbo se separa y se usa un pronombre, se da la reducción de la pronunciación del pronombre. Así es como en la oración I have to call him up el pronombre him se reduce $\mathrm{a} / \mathrm{Im} /$. Del mismo modo, se reducen los pronombres them a /əm/y her a /r/. Como primer paso, el profesor deberá enfatizar este aspecto y señalarlo por medio de cintas de audio o vídeo en donde los participantes hablen usando esa reducción. Además, el docente debe dar suficiente práctica sobre este aspecto fonológico, ya que los estudiantes tenderán a pronunciar la forma completa del pronombre, lo que redundará en un lenguaje artificial.

Esta práctica puede llevarse a cabo presentando ejemplos del verbo simple con un pronombre y luego practicar el verbo fraseológico con el pronombre. Por ejemplo, el profesor da una lista de oraciones como eat the soup, drink your milk, clean your room. Los estudiantes deben sustituir el complemento por un pronombre. Se agrega la partícula up para convertir los verbos en fraseológicos, eat the soup 
up, drink your milk up, clean your room up. Después se sustituye el complemento por un pronombre y se practica, eat it up, drink it up, clean it up. El profesor debe hacer hincapié en que dichas oraciones se pronuncian como una unidad fonológica.

Asimismo, el profesor puede hacer una serie de preguntas a las que los estudiantes deban responder con el verbo fraseológico y un pronombre. Por ejemplo:

Profesor: You have to talk to Tommy. What do you do? (Usted necesita hablar con Tommy, ¿qué hace?).

Estudiante: I call him up (Lo llamo por teléfono).

Profesor: The kitchen is really a mess. What do you have to do?

(La cocina está muy sucia. ¿Qué tiene que hacer usted?).

Estudiante: I clean it up (Tengo que limpiarla bien).

\section{Implicaciones}

De todo lo anterior se desprende la dificultad que tienen los estudiantes de habla hispana para comprender y usar este tipo de estructura del idioma inglés. Por esto, el prof esor debe considerar cada una de las diferentes características de estos verbos para así facilitarle al estudiante no sólo su comprensión sino su uso apropiado en una situación dada. Muchos expertos han dado sugerencias para facilitar su uso y comprensión. Susan Ulm sugiere que se enseñe una unidad sobre verbos fraseológicos que usen la misma partícula para indicar la misma función, por ejemplo, up, que significa "consumo total", como en eat up, drink up, use up ${ }^{10}$.

Por otra parte, el profesor necesita saber cuáles verbos son de uso más frecuente, en qué contexto se usan, cuáles de estos verbos deben separarse y cuándo deben separarse. Por lo tanto, la selección y presentación de esta estructura debe tener una base más científica

10. Susan Ulm, The Separation Phenomenon in English Phrasal Verbs (tesis inédita, UCLA, 1975). 
que la meramente subjetiva e intuitiva. A continuación, se comentan algunas sugerencias de actividades usadas en mis lecciones que han contribuido a que los estudiantes comprendan y usen los verbos fraseológicos.

\section{Algunas sugerencias metodológicas}

Mediante vídeos o grabaciones de audio los verbos fraseológicos deben ser presentados en contextos en que los interlocutores los usen para llevar a cabo diversos actos del habla. Algunas veces los estudiantes tienen la impresión de que los verbos fraseológicos son coloquiales y no deben ser usados en el lenguaje formal o escrito, lo cual es totalmente erróneo.

Los estudiantes deben aprender cada verbo como una unidad y no el verbo y su preposición o partícula por separado. El profesor debe promover el aprendizaje deductivo mediante ejemplos de los que los alumnos deben sacar una conclusión o regla. Además, la práctica de estas estructuras debe ser extensiva tanto en situaciones orales reales como en forma escrita.

La técnica de total physical response (respuesta física total) se aplica muy bien para la práctica de estas estructuras. Surgió como respuesta a métodos de enseñanza del inglés que estuvieran más relacionados con las investigaciones sicolingüísticas y con teorías sobre la adquisición de un segundo idioma. Para James Asher, creador de este método, el estudiante debía primero estar expuesto a escuchar y comprender el segundo idioma sin forzarlo a producirlo. Esto se hacía por medio de instrucciones que el profesor daba en clase y que los alumnos debían seguir. Al principio las instrucciones eran muy simples y con el desarrollo del curso se hacen más complejas. A pesar de que el método de respuesta física total no se usa mucho actualmen'te, sí se emplea como una técnica efectiva para enseñar ciertos contenidos lingüísticos. En el caso de los verbos fraseológicos, el profesor puede decir, "Go to the door, turn off the lights; then go to the 
board and cross out the word 'English', hand out a piece of paper to Juan, pick up your backpack from the floor, and put away your pencils" (Vaya a la puerta, apague las luces, vaya a la pizarra y tache la palabra 'inglés', dele un papel a Juan, recoja su mochila del suelo y guarde sus lápices). La técnica se presta muy bien para verbos tales como cut out (recortar), turn on (encender), turn off (apagar), cross out (tachar), pick out (seleccionar), hand out (entregar).

La técnica de role play (dramatizaciones) también puede servir para practicar estos verbos si se les dan a los estudiantes situaciones específicas y verbos para usar en cada una de ellas. Por ejemplo, en una conversación entre un agente de viajes y un turista se pueden usar: call up (llamar por teléfono), call off (cancelar), sign in (inscribirse), check in (reportarse en el aeropuerto u hotel), check out (salir del hotel) y fill out (llenar una solicitud).

La técnica de mixer (mezclador) consiste en dar a los estudiantes una tarea para la cual deben dar con su pareja entre sus compañeros de aula. Por ejemplo, la mitad de la clase puede recibir papeles o tarjetas con verbos fraseológicos y la otra mitad con una situación en la que el verbo se pueda utilizar adecuadamente. Los estudiantes del grupo A deben ir por el aula leyendo sus verbos hasta encontrar a los compañeros que tienen la situación que va con dichos verbos.

Ejemplo:

GRUPO A:

No, we called it off.

(No, la cancelamos.

It broke out in Hong Kong.

(Estalló en Hong Kong.

\section{GROUP B:}

So, we don't have to attend the meeting.

¿Entonces, no tenemos que ir a la reunión?)

Where did the bird flu epidemic start?

¿Adónde empezó la gripe aviaria?) 
It blew out downtown Madrid.

(Estalló en el centro de Madrid.

It broke down on the way to the party.

(Se descompuso de camino a la fiesta.

They get along just fine.

(Ellos se llevan bien.

They have to dress up.

(Tienen que vestirse de gala.

They turned it off.

(Lo apagaron.
Where did the ETA bomb explode? ¿Adónde estalló la bomba de ETA?) Where did the car malfunction? ¿Adónde se varó el carro?)

Do Tom and Liz have a good relationship?

¿Tienen Tom y Liz una buena relación?)

What kind of party is it?

¿Qué clase de fiesta es?)

What happened to the air conditioner?

¿Qué pasó con el aire acondicionado?)

La técnica de aprendizaje cooperativo promueve que los educandos trabajen en grupos y resuelvan un problema dado. En el caso de los verbos fraseológicos, esta técnica se presta muy bien para tratar este tema. Los estudiantes trabajan en grupos y se les da un sobre que contenga unos diez verbos y unas cinco partículas. Ellos deben formar tantas combinaciones como sean posibles. Después las presentan al resto de la clase.

En el área de la expresión escrita se les puede dar a los estudiantes un texto que contenga verbos corrientes y pedirles que los sustituyan por verbos fraseológicos para cambiar el tono de la expresión. Por ejemplo:

Since both Bobbyand Sue had been raised in a large urban area, they did not want their kids to have the same experience. First they boarded their car and looked around the suburbs. They also examined the Sunday paper for possible rentals in the suburbs. If they moved over to the suburbs, Sue would have to stop attending night school. They would have to discard a lot of their old furniture. Besides, Bobby 
would have to tolerate long hours in the rush-hour traffic twice a day ${ }^{11}$.

Los siguientes sitios de Internet contienen ejercicios útiles en la práctica de los verbos fraseológicos:

$$
\begin{array}{ll}
\text { - } & <\text { www.alliance.brown.ed }> \\
\text { - } & <\text { www.rit.edu }> \\
\text { - } & <\text { www.ltjournal.co.uk }> \\
\text { - } & <\text { www.daver.org }> \\
\text { - } & <\text { www.eslblue(s).com }>
\end{array}
$$

\section{Conclusión}

El inglés como idioma extranjero ha recibido gran importancia en la educación en Costa Rica. Si los programas de enseñanza tienen como uno de los objetivos primordiales el desarrollo de la habilidad comunicativa de los educandos, se debe tener en cuenta el lenguaje auténtico de los angloparlantes. Los verbos fraseológicos constituyen una estructura muy productiva en el idioma inglés. Si nuestro objetivo es enseñar a los estudiantes la competencia comunicativa, dichos verbos no deben ser dejados de lado. Por el contrario, su enseñanza y práctica deben ser incluidas a lo largo de un programa de enseñanza del inglés. Los educadores de inglés deben tener en cuenta todos los aspectos lingüísticos de los verbos fraseológicos y entender que no es una estructura fácil para los hispanoparlantes. Además, para compensar la deficiencia de muchos libros de texto, el docente debe incorporar su práctica en el desarrollo del currículo.

11. Traducción: Debido a que Bobby y Sue fueron criados en una zona urbana grande, no querían que sus hijos tuvieran una experiencia similar. Primero, se montaron al carro y mane jaron por los suburbios cercanos. También revisaron el periódico dominical para ver alquileres en los suburbios. Si se pasaban de casa, Sue tendría que dejar sus clases de noche. Tendrían que deshacerse de sus viejos muebles. Además, Bobby tendría que soportar largas horas en el tráfico de la hora pico dos veces al día. 